\title{
Acerca de la posibilidad de un currículum basado en el desarrollo cognitivo en contextos de deprivación social
}

\author{
Edita Núñez Sotelo*
}

\begin{abstract}
Resumen
El presente artículo revisa la posibilidad de un currículo basado en el desarrollo cognitivo en contextos de deprivación social, para ello se analiza primero la relación entre currículum y desarrollo cognitivo, y luego se consideran las siguientes preguntas: ¿la escuela se hace cargo del desajuste cognitivo que se produce en el estudiante a partir de su pérdida de hegemonía de información frente a los medios de comunicación?; ‘los docentes consideran e involucran en sus clases las representaciones mentales que traen los alumnos a partir de su contexto cultural, individual y social?. Ante estas interrogantes se hará una revisión crítica de las prácticas presentes en la educación en escuelas chilenas en sectores de deprivación social, con el objeto de intentar responder al planteamiento inicial sobre la posibilidad de un currículum basado en el desarrollo cognitivo en este tipo de entornos.
\end{abstract}

Palabras Clave: Currículum, Desarrollo Cognitivo, Contexto cultural, deprivación social.

\section{On the possibility of a curriculum based on cognitive development in contexts of social deprivation}

\begin{abstract}
This article overviews the possibility of developing a curriculum based on cognitive development in contexts of social deprivation. It firstly analyses the relationship between curriculum and cognitive development to then formulate the following questions: Does the school take responsibility for the cognitive imbalance produced on the student due to the loss of information hegemony before mass media? Do teachers consider and incorporate into their classrooms the mental representations students bring from their cultural, individual and social contexts? This work critically reviews current practices in Chilean schools from socially deprived areas in order to answer the initial question about the possibility of a curriculum based on cognitive development in those environments.
\end{abstract}

Key words: Curriculum; Cognitive Development; Cultural Context; Social Deprivation.

* Lic. en Psicología U. de Chile, Psicóloga U. de Chile, Magíster en Desarrollo Cognitivo mención Evaluación Dinámica de Propensión al Aprendizaje U. Diego Portales. Diplomada en Psicología y Educación mención Inteligencias Múltiples U. de Chile. Docente U. de Chile, U. Central. mail: nunez.edita@gmail.com 


\section{Introducción}

La idea de desarrollo cognitivo ha sido abordada en la escuela tradicionalmente desde una concepción estática de la inteligencia, es decir, se ha considerado que ésta es una estructura con la que nacen los sujetos y a la que la escuela le da los contenidos necesarios para el desenvolvimiento de éste en la sociedad. Como ejemplo de esto podemos recordar el antiguo dicho español "lo que natura no da, Salamanca no presta...”.

Esta concepción estática surge del paradigma conductual, el cual ha impuesto una concepción de la enseñanza desde la realidad objetiva e independiente del sujeto que aprende. El objetivo central de la escuela es proporcionar contenidos y depositar información para que el alumno la adquiera. En este contexto el concepto de inteligencia, se operacionaliza a través del concepto del coeficiente intelectual (C.I.) (Cornejo, 2003). Esta estandarización entiende la inteligencia como una característica estática e inmutable de los sujetos a través de su vida, que nace del sujeto y no tiene relación con otros factores (Spearman, 1923; Thurstone, 1938, en Nader \& Benaím, 2000; Rozas, Boetto y Jordán, 2000).

Alrededor de los años 50 comienzan a nacer críticas a este paradigma, surgiendo como respuesta el paradigma cognitivo, el cual es más flexible y más cercano al aprendiz en su situación de aprendizaje. Dentro de este paradigma la educación debiera orientarse al logro de aprendizajes significativos y con sentido y al desarrollo de habilidades estratégicas generales y específicas de aprendizaje. El enfoque cognoscitivista entiende la inteligencia como la capacidad para resolver problemas y adaptarse a situaciones nuevas adecuando el comportamiento. Su objetivo es identificar los procesos cognitivos que intervienen en la realización de las tareas o procesamiento de la información, explicando los comportamientos inteligentes como aquellos que consideran características del sujeto y del contexto. El mayor interés está en evaluar el potencial del aprendizaje de un sujeto, poniendo énfasis en que las diferencias individuales se evalúan a través de las propias actividades de procesamiento que una persona realiza. El mayor interés está en explicar las diferencias individuales y 
no en medirlas, ya que el conocimiento es construido a partir de las representaciones mentales y no se busca compararlas con un estándar objetivo, sino que meterse en cómo el sujeto construye y se implica con el objeto (Sternberg, 2003; Gardner, I983).

La educación fundada sobre la base de estos paradigmas se construye desde los discursos políticos públicos de la modernidad tendiendo a la necesidad de equidad e igualdad de oportunidades, frente a lo cual la educación y el currículo en particular juegan un rol preponderante, ya que si entendemos el currículo como "un espacio de ejercicio de poder” (Silva, 200I), es a través de tal o cual concepción de currículo como se constituye y valida una perspectiva de posible ejercicio por un lado de equidad e igualdad, como de inequidad, construcción y perpetuación de las desigualdades.

Entendiendo que un currículo basado en el desarrollo cognitivo pudiese disminuir las desigualdades en contextos de deprivación social, se plantea como objetivo de este artículo la revisión de dicha posibilidad. Se pretende identificar qué aspectos posibilitarían la integración de un currículo que apunte al desarrollo cognitivo en este tipo de establecimientos.

Para alcanzar los objetivos propuestos, este artículo desarrolla primero una revisión teórica de la relación entre currículo y desarrollo cognitivo, luego, desde la definición de lo que se entenderá como colegio en contexto de deprivación social se revisan las prácticas educativas frente a las preguntas sobre el tratamiento al desajuste cognitivo del estudiante en relación a la escuela y los medios de comunicación, y en relación a la consideración de los docentes sobre las representaciones mentales de los alumnos a partir de su contexto cultural, individual y social. Finalmente, se presentan algunas conclusiones en relación a la idea básica planteada como la posibilidad de aplicar un currículo basado en desarrollo cognitivo en entornos de deprivación social.

\section{Marco Teórico}

El objetivo de este marco teórico es presentar algunos de los planteamientos surgidos a partir de la relación entre educación 
y currículum, la cultura escolar y su relación con la sociedad y currículum y desarrollo cognitivo.

\subsection{Educación y Currículum}

Para Coll (I993), en la Educación es fundamental "el cómo se articulan lo individual y lo cultural”; esta relación determina el conjunto de actividades mediante las cuales un grupo asegura que sus miembros adquieran la experiencia social históricamente acumulada y culturalmente organizada, la cual considera los instrumentos cognitivos de naturaleza simbólica y sus usos y los procesos psicológicos superiores. Las actividades educativas adoptan diferentes modos de organización social según el volumen y también el contenido concreto del conocimiento cultural. Dentro de este contexto, el currículum se transforma en una guía, en un instrumento útil para orientar la práctica pedagógica. Esta orientación debe tener en cuenta las condiciones reales en las que se conjugarán por una parte las intenciones, los principios y las orientaciones generales, $y$ por otra, las prácticas pedagógicas.

Dentro de esta perspectiva, el currículum se construye sobre la base de cuatro premisas:

Qué enseñar

Cuándo enseñar

Cómo enseñar

Qué, cómo y cuándo evaluar

Cada uno de estos puntos se relaciona entre sí y se condicionan mutuamente, comenzando por una explicitación de las intenciones educativas (¿Qué enseñar?) hasta llegar al plan de acción de dichas intenciones (cuándo, cómo y el proceso evaluativo).

Como síntesis, Coll afirma que:

el currículum, como proyecto, preside las actividades educativas escolares, precisa sus intenciones y proporciona guías de acción adecuadas y útiles a los profesores que tienen la responsabilidad directa de su ejecución. 
Por otro lado, Gimeno Sacristán plantea que:

el currículum es el reflejo del proyecto educativo globalizador, que agrupa a diversas facetas de la cultura, del desarrollo personal y social, de las necesidades de los individuos para desenvolverse en sociedad, destrezas y habilidades consideradas fundamentales, etc.

Especial importancia releva el autor respecto de la función socializadora total que tiene la educación.

El que la función socializadora vaya más allá de los tradicionales contenidos académicos se considera normal, dada la función educativa global que se asigna a la institución escolar. [...]Desde esta perspectiva se espera que la formación básica de cualquier persona deba incluir elementos culturales cada vez más amplios, fundamentales para una educación integral. Los objetivos cada vez más extensos de las instituciones educativas básicas que involucran a todos los ciudadanos implican un currículum que comprenda un proyecto socializador y cultural también amplio (Gimeno Sacristán, 1992: 66).

Ambos autores relevan la importancia fundamental del currículum en la formación de sujetos que están en relación con su mundo. Para Coll (1993), el currículum es el espacio de articulación entre lo individual y lo social, transformándose esto en la guía para orientar la práctica pedagógica. Por su parte, Gimeno Sacristán (I992) da cuenta de la importancia de la transmisión cultural en la función socializadora de los sujetos. Esta función socializadora tiene consecuencias más allá de la escuela e implica a la cultura en general en su relación con los procesos sociales que forman al sujeto de manera global.

Gimeno Sacristán (1992) afirma que en la composición del currículum se incluye una cultura muy diversificada y con componentes valorados distintamente. Esta diversidad genera "conflictos de intereses" entre contenidos curriculares asumidos como "naturales" del currículum y otros no. Es importante señalar que esta diversidad, presente en los contenidos culturales del currículum, plantea desigualdad 
de oportunidades en la conexión entre la experiencia escolar y la extraescolar en los alumnos provenientes de diferentes contextos sociales.

Según Bourdieu (en Whitty, en G. Sacristán 1992:73) el currículum se destaca por su falta de neutralidad y que va por dos direcciones: por una parte, la opción curricular es un instrumento de diferenciación y de posible exclusión para los alumnos. En los currículum dominantes se suele pedir a todos los alumnos por igual lo que sólo unos pocos pueden cumplir. Por otra parte el currículum es un objeto no neutro especialmente en los contenidos de lenguaje, humanidades y ciencias sociales, en los que hay una peculiar dependencia y relación con el "capital cultural" que trae el alumno procedente del exterior. La enseñanza de los contenidos se realiza a través de procedimientos que continuamente dan cuenta del "capital cultural" del alumno. Importa destacar además que los componentes curriculares se proyectan en el futuro y en las aspiraciones de los diversos grupos sociales.

Es fundamental además tener claro que el estudiante no es un individuo abstracto, sino que es un sujeto que proviene de un medio social concreto y con un bagaje cultural particular que le proporciona oportunidades marcadas y un entorno específico para dar significados al currículum escolar. En este contexto se hace difícil pensar en un núcleo de contenidos curriculares obligatorios para todos y ante el cual los alumnos tengan iguales oportunidades de éxito escolar. Un currículum obligatorio debiera ser más bien la entrada, ya que por sí solo no es suficiente si no se toman en cuenta las desiguales oportunidades ante él y las adaptaciones metodológicas necesarias para favorecer la igualdad, teniendo siempre en cuenta que la escuela por sí misma no puede superar las diferencias sociales.

Muy relacionado con lo anterior, vemos que en el currículum tradicional se valora más bien la cultura de la clase media y alta (que cuentan con las competencias de saber leer, escribir, abstraer, etc.), por ello las clases menos favorecidas fracasan al encontrarse en una cultura curricular que tiene poco que ver con su entorno inmediato. Para esta diversidad de alumnos de distintos colectivos y clases sociales, el sistema curricular tiene diferentes puntos de contacto y a la vez repercute en distintas oportunidades para los diferentes grupos 
sociales, que por una cultura previa a la escuela, están en condiciones desiguales para enfrentarse al currículum.

\subsection{Cultura escolar y su relación con la sociedad}

Desde la perspectiva de Gimeno Sacristán (I992), la relación entre lo cultural-educacional y lo social tiene importantes proyecciones dentro de la institución escolar y fundamentalmente en el currículum escolar. Esto provoca que los ciudadanos se conecten por diversos medios, distintos a los escolares. Dentro de los más importantes se encuentran los medios de información, la tecnología, etc. Dentro de esta misma línea, Pérez Tornero (2000) afirma que en la sociedad actual, inmersa en la información, en las fuentes del saber y en la acumulación del conocimiento, los medios difunden las informaciones de manera muy rápida. Es en esta realidad que:

la explosión de información y el conocimiento ha rebasado el ámbito de la escuela, la cual, para mantener a los niños en un ámbito controlado de educación y aprendizaje resistente al mundo exterior ha declinado mucho. [ ] en cambio, los medios (son) los que progresivamente se encargan de proporcionar la argamasa que requiere el tejido social contemporáneo. Y lo hacen de manera que, en cierto modo, tiende a la totalización (Tornero, 2000:44).

En este contexto, Gimeno Sacristán (1992) afirma que el aprendizaje escolar no logra acercarse al aprendizaje experiencial de los alumnos, producto, entre otras cosas, de los contenidos mismos del currículum, de la rigidización de los procedimientos escolares, etc. Esta situación agranda la distancia entre la cultura de dentro y fuera de la escuela, convirtiendo a la estimulación cultural como el referente más atractivo para los alumnos.

Para el autor la disfunción entre currículum escolar e influencia exterior:

se hace más evidente para las clases medias y altas y en ambientes urbanos, con más fácil acceso a la cultura exterior, al tiempo que deben seguir inmersas en los viejos usos culturales de la escuela (Gimeno Sacristán, I992:88). 
Esta disociación entre cultura del currículum y la de los medios externos deja cada vez más obsoleta la primera. Esto trae consecuencias para los distintos alumnos. En el caso de los estudiantes de niveles culturales bajos, la cultura escolar no tiene mucha importancia en su actual vida y en sus proyectos de vida, traduciéndose esto en abandono, desmotivación, fracaso escolar y otros medios de resistencia o la rebeldía contra una institución que, por aburrida, tiene que imponerse por medio de recursos disciplinarios. Una escuela poco interesante tiene que acentuar los procedimientos represivos, inevitablemente (Gimeno Sacristán, 1992:88-89).

\subsection{Currículum y desarrollo cognitivo}

El tercer punto de nuestro análisis queremos centrarlo en el currículum y su influencia en los procesos de pensamiento.

La escuela, al hacer la disociación entre cultura escolar y el aprendizaje experiencial del alumno (Gimeno Sacristán, 1992), no considera las formas de representación que los seres humanos utilizan en sus procesamientos y cogniciones. Según E. Eisner.

las formas de representación que se usan como sistemas de símbolos para representar lo que se piensa influyen en los procesos de pensamiento y en los productos de éste, desarrollan distintas destrezas cognoscitivas e inciden en lo que uno es capaz de ver $y$ de representar.

Según el autor, estas formas de representación se encuentran presentes en la cultura, siendo la mente

un logro cultural cuya conformación está influida significativamente por el tipo de experiencias vivenciadas por una persona en el transcurso de su vida.

(Eisner, 1998:I)

La mente, como producto cultural, se va formando a través de las experiencias del sujeto. De esta manera su conformación y capacidad se ve fuertemente influida por lo que a los sujetos se les da la posibilidad de aprender. En este sentido el currículum se transforma 
en un elemento poderoso para influir la mente de los alumnos. Las políticas públicas y los maestros elaboran (en su proyecto curricular) aquello a que los niños tendrán acceso, dando forma a la clase o categoría de mentes que están construyendo. Esto trae consecuencias en la forma que la cultura (en la que todos estamos insertos) adquirirá.

La representación mental implica el desarrollo de diferentes destrezas cognitivas. La influencia del currículum en este sentido es fundamental ya que éste determina qué deben aprender a pensar los niños. Esta influencia no sólo se queda en la representación sino que luego, influye en lo que uno es capaz de ver. En este sentido, el currículum como determinante de las representaciones de los alumnos no es una herramienta neutral ya que su influencia actuará como un molde que determinará que el alumno busque lo que sabe encontrar y encuentre lo que sabe hacer. De esta manera, el aporte curricular influirá en los grados de expansión de la mente y en la construcción, que los alumnos realizan del mundo.

\section{Análisis y Discusión}

A continuación describiremos lo que entendemos por contextos escolares socialmente deprivados.

En estas escuelas el origen de los estudiantes es de pobreza, con grandes carencias económicas, problemas de cesantía, alimentación y en muchos casos con familias desestructuradas. El nivel escolar de los padres es bajo, por lo tanto existen dificultades para involucrarse en la escolaridad de los niños, además las problemáticas familiares suelen impactar directamente en la escuela sin mayor mediación desde la familia.

Con respecto a los profesores o equipos de profesores, estos subestiman las posibilidades de aprendizaje y desarrollo cognitivo y afectivo de los alumnos. Desde una perspectiva psicologizante justifican los malos resultados académicos como algo intrapsíquico y/o intrafamiliar del sujeto, y donde el colegio no tiene posibilidad de intervenir, estableciendo los problemas en un nivel estructural, es decir, se entienden como aspectos integrados al orden mental de los sujetos y que no tienen modificabilidad. 
Los alumnos por su parte asumen como propias las representaciones que surgen de los equipos docentes hacia ellos, es decir, comparten la idea de limitación como una "autolimitación" en los planos afectivo, cognitivo y social, apropiándose de la idea de no modificabilidad en estos mismos planos.

En este contexto el discurso educativo apunta a dar importancia a: aprendizaje significativo, experiencias previas y las representaciones mentales que construyen los alumnos inmersos en la actividad de enseñanza y aprendizaje.

El planteamiento de estos conceptos es muy impreciso, ya que es posible dar más de una interpretación a dichos conceptos. Estas posibles interpretaciones, incluso pueden ser contradictorias en relación al fondo del planteamiento teórico original. Por ejemplo, docentes que afirman que "antes de enseñarles a multiplicar veo sus conocimientos previos y les pregunto si saben sumar...”. En este caso se confunden los contenidos teóricos que el alumno debe manejar de manera previa, con los conocimientos individuales, sociales y culturales propios del entorno de origen del sujeto.

En la práctica los conceptos a los cuales hacemos alusión fortalecen un enfoque conductual, es decir, se enfatiza resultados y rendimiento, priorizando la acción del profesor de pasar los contenidos sin considerar los procesos de aprendizaje, de pensamiento y desarrollo de las capacidades necesarias para adquirir esos conocimientos. Quien no logra aprender en estas condiciones, no tiene posibilidades de adquirir mayores conocimientos.

Por otro lado, los docentes se ven presionados por los criterios que maneja el sistema de evaluación que privilegia productos y rendimientos. Así, el profesor cuando no logra los aprendizajes esperados en la cantidad de alumnos que le exige el sistema, falsea el porcentaje de logro de fijación de la nota mínima de aprobación, con lo cual, alumnos que no debieran ser aprobados por no haber logrado los aprendizajes, aparecen como aprobados.

Dentro de todo este panorama, la concepción de enseñanza que se ejerce en las escuelas se basa en una transmisión de contenidos que realiza un agente instruccional (el profesor), sin realizar mediación 
de dichos contenidos. Con esto un número importante de alumnos avanza en los niveles de estudio pero no avanza en aprendizaje, lo cual se traduce en reproducción de la desigualdad en las escuelas y liceos insertos en contextos de deprivación social.

Junto con lo anterior, y en relación al componente cultural se sostiene que las dificultades de aprendizaje que no permiten una enseñanza en condiciones de igualdad se deben a las condiciones estructurales intrapsíquicas e intrafamiliares que los niños traen, desligándose de responsabilidad a los profesores y a la escuela, y a la vez, haciendo manifiesta la representación de la inteligencia como algo estático, no dando cabida a una modificabilidad cognitiva estructural.

El currículum es fundamentalmente desarrollado desde una mirada dominante, donde los contenidos se estandarizan para todos los niños sin considerar su capital cultural de origen, su proceso de socialización y todo el contenido experiencial que el alumno puede tener acumulado. Al vivir los niños día a día esta práctica se enfrentan al conflicto de tener que adaptar sus experiencias de la vida cotidiana, que se ven marcadas por el acceso a medios de comunicación como radio, periódicos, y sobre todo la televisión, etc. El acceso a estos medios y su no incorporación dentro del espacio escolar provoca en este contexto una rigidización y una reafirmación de un currículum dominante, que tiende a la uniformidad, donde todos deben rendir en lo que se les pida, sin atender a la diversidad. De esta manera, la escuela no se haría cargo del desajuste cognitivo que se produce en el alumno producto de la pérdida de hegemonía de información de la escuela frente a los medios, ya que no incorpora la cultura que se transmite a través de los medios de comunicación y a la cual los alumnos tienen acceso, tanto desde un punto de vista de las estructuras de los medios, que debieran ser incorporados a través del currículum, como de la posibilidad de negociar los contenidos culturales del currículum con los contenidos culturales presentes en los medios de comunicación de masas.

En consecuencia, el cómo los alumnos integran su propia cultura dentro de su aprendizaje, implica para la escuela un desafío que no siempre se recibe como tal y donde esto se vivencia como una carga, una tarea impuesta a la escuela por la sociedad. 
Otra consecuencia importante que aparece tras producirse esta disociación entre la propia cultura y la cultura escolar se refiere a las representaciones mentales que los niños construyen y que no siempre encuentran espacio para presentarse y madurar dentro de la escuela. En el contexto escolar la formación de la mente no es vista como un objetivo a lograr, la idea que está más bien en la base es la de un "libro abierto" donde la experiencia escolar se va plasmando, de esta manera las representaciones mentales se circunscriben a este currículum dominante presente en la escolarización, que indica que los niños deben aprender a reproducir determinados contenidos, que muchas veces no se enriquecen con las experiencias previas ni con sus propios contextos culturales y donde se tiende más bien a la uniformidad y no a la expansión del artefacto cultural de la mente, más bien, en la práctica el currículum apunta a la creación de productos que son finalmente evaluados, sin considerar la elaboración cognitiva y afectiva que existe detrás. En este punto cabe preguntarse qué rol juega el Ministerio de Educación, que si bien declara que la educación chilena se mueve en el ámbito discursivo en un nivel más cognitivo y constructivista, en las prácticas pedagógicas y sobre todo en la evaluación, se rige por un modelo más bien conductual. Las evaluaciones nacionales apuntan a evaluar manejo de contenido y no la adquisición de competencias cognitivas y construcción de conocimientos en el alumno. Así la escuela privilegia el entrenamiento para lograr altos puntajes de rendimiento en la prueba SIMCE, ya que esto la valida dentro del sistema escolar y social.

\section{Conclusiones}

Nos debe quedar claro que a través de las actuales prácticas pedagógicas es muy poco probable realizar una intervención en desarrollo cognitivo en términos globales o institucionales dentro de un establecimiento educativo inserto en un contexto de deprivación social.

Las razones esgrimidas a partir de las respuestas a nuestras dos preguntas iniciales apuntan claramente a que por un lado la escuela no se hace cargo del desajuste cognitivo que se produce en el estudiante a partir de la pérdida de hegemonía de la información de ésta frente 
a los medios de comunicación, ni tampoco considera e incorpora las representaciones mentales de los alumnos a partir de su contexto individual, social y cultural.

La posibilidad de plantearnos un currículum basado en el desarrollo cognitivo dentro de un contexto de deprivación social requiere al menos, la consideración de los dos puntos señalados en la discusión, es decir, al incorporar los contenidos culturales de los medios de comunicación junto con la cultura de origen de los alumnos se genera una apertura cognitiva, ya que, al producirse la incorporación de valores presentes en la cultura cotidiana se humaniza la relación entre profesor y estudiante, cambiando el rol de objeto a sujeto de los niños y jóvenes insertos en escuelas y liceos en contextos de deprivación social.

\section{Bibliografía}

Coll, C. (1993). Psicología y currículum: una aproximación psicopedagógica a la elaboración del currículum escolar. Barcelona: Paidós.

Cornejo, R. (2003). “'Se puede medir la inteligencia? El coeficiente intelectual y el desarrollo del pensamiento”. Boletín Mediar n ${ }^{\circ}$ I5. Santiago: Centro de Desarrollo Cognitivo. Universidad Diego Portales.

Eisner, E. (I998). “Cognición y representación: persiguiendo un sueño”. Revista Enfoques Educacionales Vol.I Nº I, I998. Departamento de Educación, Facultad de Ciencias Sociales. Universidad de Chile.

Gardner, H. (I983). Estructuras de la Mente. La teoría de las inteligencias múltiples. Acceso el I de abril de 20II: disponible en http://epistemologiadoctoradounermb.bligoo.es/media/users/I6/8I2365/ files/I $42090 /$ ESTRUCTURAS_DE_LA_MENTE_-_ Howard_Gadrner.pdf

Gimeno,J. (I992). El currículum: una reflexión sobre la práctica. Madrid: Morata.

Nader, M. \& Benaím, D. (2000). "La Inteligencia de los Niños”. Psicodebate 5. Psicología, cultura y sociedad. Acceso el 15 de marzo de 201 I: disponible en www.palermo.edu/.../pdf/.../5Psico\%2002.pdf

Pérez Tornero, J. (2000). Las escuelas y la enseñanza en la sociedad de la información. Barcelona: Paidós. 
Rozas, R., Boetto C. y Jordán V. (2000). Introducción a la Psicología de la Inteligencia. Textos universitarios. Facultad de Ciencias Sociales. Santiago: Pontificia Universidad Católica de Chile.

Silva, M. (200I). "Cambio y Difusión Curricular. Aspectos centrales". Revista Enfoques Educacionales - Volumen 3 Número I, 2000-200I. Departamento de Educación. Facultad de Ciencias Sociales. Universidad de Chile. Acceso el 8 de mayo de 20II. Disponible en http://www. facso.uchile.cl/publicaciones/enfoques/06/docs/enfoques_06_2000. pdf

Sternberg, R. y Grigorenko, E. (2003). Evaluación dinámica. Naturaleza y medición del potencial de aprendizaje. Barcelona: Paidós. 